\title{
Article \\ Mutualism between Gut-Borne Yeasts and Their Host, Thaumatotibia leucotreta, and Potential Usefulness in Pest Management
}

\author{
Marcel van der Merwe ${ }^{1,2} *(\mathbb{D})$, Michael D. Jukes ${ }^{1,2} \mathbb{D}$, Caroline Knox ${ }^{1}$, Sean D. Moore ${ }^{2,3}$ (D) and Martin P. Hill ${ }^{2}$ (D) \\ 1 Department of Biochemistry and Microbiology, Rhodes University, P.O. Box 94, Makhanda 6140, South Africa; \\ m.jukes@ru.ac.za (M.D.J.); caroline.knox@ru.ac.za (C.K.) \\ 2 Centre for Biological Control, Department of Zoology and Entomology, Rhodes University, P.O. Box 94, \\ Makhanda 6140, South Africa; seanmoore@cri.co.za (S.D.M.); m.hill@ru.ac.za (M.P.H.) \\ 3 Citrus Research International, P.O. Box 5095, Walmer, Gqeberha 6013, South Africa \\ * Correspondence: marcelvd.merwe93@gmail.com
}

Citation: van der Merwe, M.; Jukes, M.D.; Knox, C.; Moore, S.D.; Hill, M.P. Mutualism between Gut-Borne Yeasts and Their Host, Thaumatotibia leucotreta, and Potential Usefulness in Pest Management. Insects 2022, 13, 243. https://doi.org/10.3390/ insects13030243

Academic Editor: Bessem Chouaia

Received: 10 January 2022

Accepted: 10 February 2022

Published: 28 February 2022

Publisher's Note: MDPI stays neutral with regard to jurisdictional claims in published maps and institutional affiliations.

Copyright: (C) 2022 by the authors. Licensee MDPI, Basel, Switzerland. This article is an open access article distributed under the terms and conditions of the Creative Commons Attribution (CC BY) license (https:// creativecommons.org/licenses/by/ $4.0 /)$.
Simple Summary: The false codling moth is an indigenous pest of the citrus industry in southern Africa. It is a major threat as it can cause substantial fruit damage through larval feeding. The microorganisms associated with insects are often studied for their potential to kill their host instead of how they could aid them. However, in recent years, this aspect of their interaction has received much attention. The codling moth has been shown to have a close relationship with the naturally occurring yeast found within their digestive tract. These beneficial yeasts help the adult female moths locate a suitable host for egg laying. During their larval phase, these yeasts help them develop faster and increase their chance of survival. These interactions could be manipulated for the purposes of biological control, as one might be able to combine these yeasts with existing biological control agents to improve their effectiveness. These yeasts may also provide additional methods for monitoring field populations of insect pests. In this study, we identified yeasts that occur naturally in the guts of false codling moth larvae and investigated whether any of them are beneficial to their host.

Abstract: Thaumatotibia leucotreta is endemic to southern Africa and is highly significant for various fruit industries, including the South African citrus industry, due to its classification as a phytosanitary pest. Mutualistic associations between C. pomonella, closely related to T. leucotreta, and yeasts have previously been described and reported to reduce larval mortality and enhance larval development. Here, we determined which yeast species occur naturally in the gut of T. leucotreta larvae and investigated whether any of the isolated yeast species affect their behaviour and development. Navel oranges infested with $T$. leucotreta larvae were collected from geographically distinct provinces in South Africa, and the larvae were processed for analysis of naturally occurring associated yeasts. Six yeast species were isolated and identified from the guts of these T. leucotreta larvae via PCR amplification and sequencing of the ITS region of rDNA and D1/D2 domain of large ribosomal subunit. Larval development and attraction assays were conducted, and T. leucotreta larvae that fed on Navel oranges inoculated with yeast had accelerated developmental periods and reduced mortality rates. Neonate T. leucotreta were also attracted to YPD broth cultures inoculated with yeast for feeding. Oviposition preference assays were conducted with adult T. leucotreta females. Navel oranges inoculated with yeast were shown to influence the oviposition preference of adult females. Yeasts harbour the potential for use in biocontrol, especially when combined with other wellestablished control methods. This study provides a platform for future research into incorporating yeast with current biological control agents as a novel option for controlling T. leucotreta in the field.

Keywords: insect-microbe interaction; mutualism; false codling moth; yeast 


\section{Introduction}

Microbes are the most abundant living organisms on earth, and apart from being found within the general environment, they are also known to colonise other living organisms such as insects [1]. The relationships between insects and microorganisms are often not overtly pathogenic but rather beneficial or even required [2]. They vary considerably and range from accidental encounters to functional ones, such as locating an attractive food source or providing essential nutrients missing from their diet [3,4]. Interactions between insects and microbes with a mutualistic relationship are greater than those that interact by chance, as they have evolved together $[5,6]$. Microbes are predominantly localised to the external cuticle, gut, hemocoel and cells of insects, as these regions offer the most favourable conditions for establishment [1]. Microbes ingested and hosted within the insect gut can strongly impact insect survival $[7,8]$. The makeup of these microbial communities mainly comprise bacteria, archaea and eukaryotic microorganisms $[9,10]$. The microbiota harboured within insects is generally distinct from the microorganisms in the external environment [2]. Microbial populations are usually concentrated in the digestive tract of most insects, particularly the hindgut, as it is a benign environment that provides access to nutrients and protection from the various stresses associated with the external environment [3]. The role of microbial communities in insect-plant interactions is essential and can favourably impact their ecology, evolution and behaviour [11-15]. Several insect orders have been reported to harbour persistent microbial communities [16]. Associations between Lepidoptera and microbes have rarely been described even though they are the second-most diverse insect order with some of the most devastating agricultural pests worldwide [17,18].

Unlike their bacterial counterparts, interactions between insects and yeasts have been understudied, despite their importance to insect fitness and behaviour $[9,19,20]$. The search for associations between Lepidoptera and yeasts was ignited by studies conducted on Drosophila melanogaster (Meigen) (Diptera: Drosophilidae) [21]. The presence, type and amount of yeast within their diet affect their behaviour, development and fitness $[14,22,23]$. Yeasts were also shown to be primarily responsible for influencing host locating and ovipositing, not plant cues as once thought [24]. Yeasts have proven to be an essential nutritional source for insect larval development and to influence neonate larval feeding and behaviour [25]. For Cydia pomonella (Linnaeus) (Lepidoptera: Tortricidae), commonly known as the codling moth, larvae are closely associated with yeast from the genus Metschnikowia [26]. These yeasts were reported to be crucial in enhancing the development of $C$. pomonella larvae and reducing mortality. Additionally, the metabolites produced by yeasts contributed towards adult C. pomonella moths recognising and finding their host plant [26]. Identifying this mutualistic association between C. pomonella larvae and yeasts from the genus Metschnikowia has led to the development of novel biocontrol strategies [27-29].

Thaumatotibia leucotreta (Meyrick) (Lepidoptera: Tortricidae), commonly known as the false codling moth, is a phytophagous insect, endemic to southern Africa [30]. It is important to various fruit industries, including the South African citrus industry, due to its classification as a phytosanitary pest by several international markets [31]. The pest is capable of causing significant financial loss through larval feeding. However, in recent years, it has been effectively suppressed, through much improved management [32-34]. There are several biological control agents available for use against T. leucotreta in South Africa $[33,35]$. Most notably, however, is the betabaculovirus, Cryptophlebia leucotreta granulovirus (CrleGV), which is an effective microbial control agent against T. leucotreta [36] It has previously been demonstrated, in laboratory assays and field trials, that combining yeast with baculoviruses has resulted in the significant increase in larval mortality $[27,28]$. This indicates that yeasts harbour the potential for use in biocontrol, especially when combined with other well-established control methods. This has, however, only been reported in C. pomonella and not for T. leucotreta. 
Herein, we aimed to determine which yeast species occur naturally in the gut of T. leucotreta larvae from geographically distinct citrus-producing regions across South Africa and to examine whether any isolated yeast species affected the behaviour and development of neonate larvae, as well as the oviposition preference of adult female moths. These yeasts could potentially represent rich resources of new biological agents for the control of T. leucotreta when combined with CrleGV.

\section{Materials and Methods}

\subsection{Yeast Isolation and Purification}

Ten Navel oranges (Citrus sinensis L. Osbeck) infested with T. leucotreta larvae were collected from one orchard in each of three geographically distinct citrus-producing regions in South Africa, namely Stellenbosch (Western Cape), Addo (Eastern Cape) and Nelspruit (Mpumalanga), between March and June of 2018 (Figure 1).

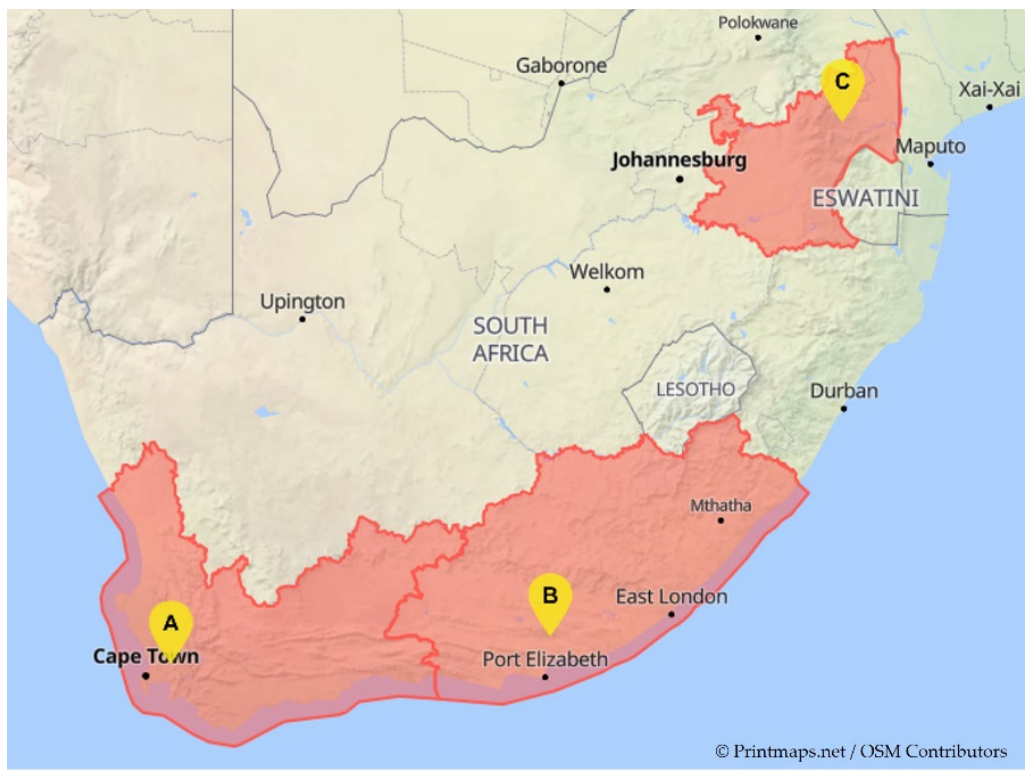

Figure 1. Citrus-producing regions across South Africa where T. leucotreta larvae were collected: (A) Stellenbosch in Western Cape, (B) Addo in Eastern Cape and (C) Nelspruit in Mpumalanga.

The extracted larvae $(n=10)$ were surface-sterilised by rinsing in $10 \% \mathrm{NaOCl}$, followed by a rinse in $70 \%$ ethanol solution $(v / v)$ and finally double-distilled $\mathrm{H}_{2} \mathrm{O}\left(\mathrm{ddH}_{2} \mathrm{O}\right)$. Yeasts were isolated from the digestive tract of T. leucotreta larvae according to Witzgall et al. [26]. Larvae were stored at $4{ }^{\circ} \mathrm{C}$ for 2 hours (h) to immobilise them. They were then dissected with a sterile razor blade in a fume hood. A sterilised inoculating loop was used to collect the gut content, which was then homogenised with a glass rod in an Eppendorf tube. The homogenate was then streaked onto a Yeast extract Peptone Dextrose (YPD) agar (SigmaAldrich, St. Louis, MO, USA) plate containing 40 units $/ \mathrm{mL}$ of penicillin (Pen) and $40 \mu \mathrm{g} / \mathrm{mL}$ of streptomycin (Strep) (Thermo Fisher Scientific, Waltham, MA, USA). Additionally, the homogenate was diluted in $\mathrm{ddH}_{2} \mathrm{O}$, filtered through muslin cloth and spread onto a Pen/Strep YPD agar plate. Plates were incubated at $27^{\circ} \mathrm{C}$ for $48 \mathrm{~h}$ under aerobic conditions, with emerging yeast colonies being selected and discontinuously streaked on Pen/Strep YPD agar plates. This process was conducted twice to obtain pure colonies.

\subsection{Yeast Identification}

Yeast isolates were grown overnight in YPD medium containing 40 units $/ \mathrm{mL}$ of Pen and $40 \mu \mathrm{g} / \mathrm{mL}$ of Strep at $27^{\circ} \mathrm{C}$ while shaking $(180 \mathrm{rpm})$. Genomic DNA (gDNA) was extracted using the YeaStar ${ }^{\mathrm{TM}}$ Genomic DNA Kit (Zymo Research, Irvine, CA, USA) per the manufacturer's instructions. The concentration of gDNA was determined using a 
NanoDrop 2000 spectrophotometer (Thermo Fisher Scientific, Waltham, MA, USA). The amplicons of the intended targeting region were amplified using $50 \mathrm{ng}$ of gDNA template with the site-specific primers (Table S1) for the internal transcribed spacer (ITS) region and D1/D2 domain of the LSU of ribosomal DNA (rDNA) [37]. PCR amplification was carried out using a SimpliAmp ${ }^{\mathrm{TM}}$ Thermal Cycler (Thermo Fisher Scientific, Waltham, MA, USA) under the following cycle parameters: $94{ }^{\circ} \mathrm{C}$ for $5 \mathrm{~min}$ followed by 30 cycles of $95{ }^{\circ} \mathrm{C}$ for $30 \mathrm{~s}, 55^{\circ} \mathrm{C}$ for $45 \mathrm{~s}$ and $72{ }^{\circ} \mathrm{C}$ for $45 \mathrm{~s}$. A final elongation cycle of $72{ }^{\circ} \mathrm{C}$ for $10 \mathrm{~min}$ was used [26]. Amplicons were sequenced by Inqaba Biotechnical Industries (Pty) Ltd. (Johannesburg South Africa). Sequences were assembled and analysed in MEGA X [38], with any ambiguous nucleotides being corrected before the sequences were identified by comparison to the GenBank database of nonredundant sequences using BLAST.

\subsection{Thaumatotibia leucotreta Culture}

Eggs and pupae were obtained from the heterogeneous T. leucotreta culture, known as "Mixed Colony", held at Rhodes University's Department of Zoology and Entomology, South Africa. The colony was established in 1996 using T. leucotreta collected from Citrusdal (Western Cape Province), Zebediela (Limpopo Province) and the Eastern Cape [39]. The larvae were reared and maintained on an artificial diet consisting of maize meal, wheat germ, casein, Brewer's yeast, nipagin, sorbic acid and distilled water [40].

\subsection{Larval Development Assays}

A modified version of the larval development assay described by Witzgall et al. [26] was used to determine the effect that yeasts had on the development of T. leucotreta larvae. Navel oranges were collected from orchards in the Sundays River Valley in the Eastern Cape Province of South Africa and stored in a $4{ }^{\circ} \mathrm{C}$ cold room to preserve the fruit until use. Oranges were sterilised in a $0.5 \%$ bleach solution, rinsed twice in $\mathrm{ddH}_{2} \mathrm{O}$ and allowed to air dry in a controlled-environment (CE) room at $25^{\circ} \mathrm{C}$ overnight. Yeast cultures were grown in YPD medium containing $40 \mathrm{units} / \mathrm{mL}$ of Pen and $40 \mu \mathrm{g} / \mathrm{mL}$ of Strep for $20 \mathrm{~h}$ at $27^{\circ} \mathrm{C}$ while shaking; cell counts were adjusted to $2 \times 10^{6}$ cells $\mathrm{mL}^{-1}$ with $\mathrm{ddH}_{2} \mathrm{O}$. Sterilised Navel oranges were dipped three times into a specific yeast culture, placed on a platform and allowed to air-dry in a $25^{\circ} \mathrm{C} \mathrm{CE}$ room. Five newly hatched T. leucotreta neonates were then placed onto each Navel orange and maintained in a $25{ }^{\circ} \mathrm{C} \mathrm{CE}$ room for 35 days (d). Cotton wool was placed between the Navel oranges $10 \mathrm{~d}$ later to provide emerging 5 th instars with a pupation site. Thereafter, the cotton wool was checked daily, and the number of pupated T. leucotreta larvae was recorded. After $35 \mathrm{~d}$, each Navel orange was dissected to check for any remaining T. leucotreta larvae. Assays were conducted on four dates, with nine Navel oranges per treatment.

\subsection{Larval Feeding Assay}

Two-choice bioassays were used to assess neonate T. leucotreta yeast feeding and behaviour, adapted from Ljunggren et al. [41]. Yeast cultures were grown as previously described, with cell counts adjusted to $1.5 \times 10^{7}$ cells $\mathrm{mL}^{-1}$ with $\mathrm{ddH}_{2} \mathrm{O}$. Two-choice bioassays were conducted whereby two $50 \mu \mathrm{L}$ drops of yeast culture and YPD medium were pipetted across from one another, approximately $1 \mathrm{~cm}$ from the edge of a plastic petri dish Red and blue colourants (Pioneer Foods, Paarl, South Africa) were used at 1:25 dilution to colour the yeast and YPD medium to distinguish between neonate T. leucotreta that fed on yeast culture, blank medium, or both. Preliminary tests did not show a bias in neonate T. leucotreta attraction to either the red or blue colourants (Figure S1) $(p>0.05 ; \mathrm{t}=0.2133$; $\mathrm{df}=27$ (Student's $t$-test)). Neonate T. leucotreta were starved for 24 to $48 \mathrm{~h}$ after hatching before being placed in the centre of a petri dish using a fine paintbrush. A glass lid was used to cover the petri dish to prevent the neonates from escaping and was left for $2 \mathrm{~h}$ to allow the larvae to feed. The colouration of the neonate's gut was then observed under a dissecting microscope. Twenty-two independent replicates with $10 \mathrm{~T}$. leucotreta neonates were performed for each yeast. 


\subsection{Oviposition Preference Assays}

A modified version of the ovipositional preference trials described by Love et al. [42] was used to determine the oviposition preference of adult T. leucotreta females. Thaumatotibia leucotreta female and male pupae were kept in separate vials. Once moths had eclosed, they were paired together, within $24 \mathrm{~h}$, and allowed to copulate for $48 \mathrm{~h}$ in a $25 \mathrm{~mL}$ glass vial, plugged with cotton wool moistened in a $10 \%$ sugar water solution. Pairs were kept in a $25^{\circ} \mathrm{C} \mathrm{CE}$ room with $30-60 \%$ relative humidity $(\mathrm{RH})$ under light conditions. Trials were conducted in a $25{ }^{\circ} \mathrm{C}$ CE room with 30-60\% RH under a day/night light cycle of $12 \mathrm{~h}$ of light and $12 \mathrm{~h}$ of dark. Navel oranges were collected and stored as previously described and removed from cold storage $1 \mathrm{~d}$ prior to being used in the ovipositional preference trial. The fruits were checked for T. leucotreta eggs, those with eggs being discarded, before being thoroughly washed in a $0.5 \% \mathrm{NaOCl}$ solution, rinsed twice in $\mathrm{ddH}_{2} \mathrm{O}$ and allowed to air dry in a CE room at $25{ }^{\circ} \mathrm{C}$ overnight. Yeast cultures were grown as previously described, with counts adjusted to $2 \times 10^{6}$ cells $\mathrm{mL}^{-1}$ with $\mathrm{ddH}_{2} \mathrm{O}$. Sterilised Navel oranges were dipped three times into a specific treatment and allowed to air-dry in a $25{ }^{\circ} \mathrm{C} \mathrm{CE}$ room. Treated Navel oranges were then placed onto $4 \mathrm{~cm}$ tall plastic platforms $15 \mathrm{~cm}$ apart in a plastic container $(60 \times 40 \times 40 \mathrm{~cm})$ covered with muslin cloth. A single pair of mated adults was then released into the container. The eggs oviposited on the Navel oranges were counted after $48 \mathrm{~h}$. Ten ovipositional preference trials were conducted per yeast species.

\subsection{Statistical Analysis}

All statistical analyses were performed using GraphPad Prism version 9.0.0 (GraphPad Software, San Diego, CA, USA). Thaumatotibia leucotreta larval development assays were analysed using a Fisher's exact test [26]. The larval feeding of T. leucotreta on yeasts was compared using a Student's $t$-test, with the level of significance set to $p=0.05$ [41]. The ovipositional preference trials were analysed using a Student's $t$-test, with a significance level of $p=0.05$ [42].

\section{Results}

\subsection{Yeast Isolation and Identification}

The guts of $30 \mathrm{~T}$. leucotreta larvae were screened for yeast, which resulted in the isolation of six yeast species. The isolated yeast species were successfully identified via the gene sequence analysis of the ITS region and D1/D2 domain of the LSU (Table 1). The occurrence of the isolated yeast species was not consistent, and the isolated species were not uniformly found in all larvae.

Three yeast species were isolated only from T. leucotreta larvae collected from Addo, viz. Meyerozyma guilliermondii, Hanseniaspora uvarum and Clavispora lusitaniae. Kluyveromyces marxianus was only isolated from a larva collected in Stellenbosch, and the remaining two yeast species, Pichia kudriavzevii and P. kluyveri, were common in larvae collected from each region.

\subsection{Larval Development Assays}

Navel oranges treated with yeast significantly decreased the larval development period and increased the larval pupation rate compared to sterilised fruit with no yeast treatment (Table 2). Pichia kluyveri $(p=0.0001), H$. uvarum $(p=0.0092)$ and S. cerevisiae $(p=0.0024)$ performed the best out of all the applied yeast treatments, as not only was development significantly faster than that of the control at a $99 \%$ probability level, but pupation success was highest for these three species. Although P. kudriavzevii $(p=0.0586)$, K. marxianus ( $p=0586)$ and C. Iusitaniae $(p=0.002)$ also significantly decreased the larval development period at the same probability level, the number of pupated larvae was similar to that of the control. Additionally, although not recorded, it was noted that the occurrence of mould was lower on yeast-inoculated Navel oranges compared to the untreated control. 
Table 1. BLAST analysis of the ITS region and D1/D2 domain for each isolated yeast species.

\begin{tabular}{ccccc}
\hline Sample & Amplicon & Yeast Species & Accession Number & Identity \\
\hline \multirow{2}{*}{ Addo “One" } & ITS & Meyerozyma guilliermondii & MN537824.1 & $100 \%$ \\
& D1/D2 & Meyerozyma guilliermondii & EU285513.1 & $100 \%$ \\
\hline \multirow{2}{*}{ Addo "Two" } & ITS & Hanseniaspora uvarum & MN371907.1 & $98.28 \%$ \\
& D1/D2 & Hanseniaspora uvarum & AY305681.1 & $99.64 \%$ \\
\hline Addo & ITS & Clavispora lusitaniae & KP765042.1 & $99.52 \%$ \\
"Three" & D1/D2 & Clavispora lusitaniae & MG871742.1 & $99.82 \%$ \\
\hline \multirow{2}{*}{ Stellenbosch } & ITS & Kluyveromyces marxianus & KX376261.1 & $99.70 \%$ \\
& D1/D2 & Kluyveromyces marxianus & CP009307.2 & $100 \%$ \\
\hline Common & ITS & Pichia kluyveri & KM982973.1 & $99.75 \%$ \\
“One" & D1/D2 & Pichia kluyveri & MN464128.1 & $99.64 \%$ \\
\hline Common & ITS & Pichia kudriavzevii & LC389027.1 & $100 \%$ \\
"Two" & D1/D2 & Pichia kudriavzevii & MF461295.1 & $100 \%$ \\
\hline
\end{tabular}

Table 2. The pupation rate of T. leucotreta larvae before or after $25 \mathrm{~d}$, during $35 \mathrm{~d}$ detached fruit bioassays. Sterilised Navel oranges $(n=36)$ were inoculated with yeast or left untreated. * and ${ }^{* *}$ indicate significant differences from the control according to a Fisher's exact test $(p<0.05$ and $p<0.01$, respectively).

\begin{tabular}{cccccc}
\hline \multirow{2}{*}{ Treatments } & \multirow{2}{*}{ Total Pupated } & \multicolumn{2}{c}{ Pupated } & Percentage & \multirow{2}{*}{-Value } \\
& & Before 25 d & After 25 d & before 25 d & \\
\hline Control & 41 & 15 & 26 & $37 \%$ & \\
M. guilliermondi & 59 & 35 & 24 & $59 \%$ & $0.0414^{*}$ \\
P. kluyveri & 78 & 58 & 20 & $74 \%$ & $0.0001^{* *}$ \\
P. kudriavzevii & 58 & 35 & 23 & $60 \%$ & $0.0253^{*}$ \\
H. uvarum & 63 & 40 & 23 & $63 \%$ & $0.0092^{* *}$ \\
C. lusitaniae & 42 & 30 & 12 & $71 \%$ & $0.0020^{* *}$ \\
K. marxianus & 58 & 38 & 20 & $66 \%$ & $0.0075^{* *}$ \\
S. cerevisiae & 62 & 42 & 20 & $68 \%$ & $0.0024^{* *}$ \\
\hline
\end{tabular}

The mortality rate of $T$. leucotreta larvae was significantly lower on Navel oranges treated with $M$. guilliermondii $(p=0.0452)$, P. kluyveri $(p<0.0001)$, H. uvarum $(p=0.0144)$ and S. cerevisiae ( $p=0.0194)$ (Figure 2). Clavispora lusitaniae $(p>0.9999), P$. kudriavzevii $(p=0.0586)$ and K. marxianus $(p=0.0586)$ did not decrease larval mortality. Overall, $P$. kluyveri provided the most benefit to T. leucotreta larvae, as the yeast decreased larval mortality by $20.55 \%$ and $74 \%$ of larvae pupated before $25 \mathrm{~d}$.

\subsection{Larval Feeding Assay}

The feeding preference of neonate T. leucotreta was influenced by four yeasts, viz. P. kluyveri $(p=0.0043)$, H. uvarum $(p=0.0037)$, P. kudriavzevii $(p=0.0001)$ and K. marxianus $(p=0.0005)$ (Figure 3). Two yeast species, M. guilliermondii $(p=0.0606)$ and S. cerevisiae $(p=0.2579)$, had no significant effect on larval feeding. The only time larvae preferred the YPD medium was in the case of $C$. lusitaniae ( $p=0.5907)$.

\subsection{Oviposition Preference Assays}

Significantly more eggs were oviposited on Navel oranges inoculated with $M$. guilliermondii $(p=0.0090), P$. kudriavzevii $(p=0.0471)$ and H. uvarum $(p=0.0013)$ compared to the untreated control fruit during the two-choice tests (Figure 4). Pichia kluyveri $(p=0.3768), C$. lusitaniae $(p=0.5729), K$. marxianus $(p=0.2838)$ and S. cerevisiae $(p=0.0517)$ did not influence the oviposition preference of adult $T$. leucotreta females. 


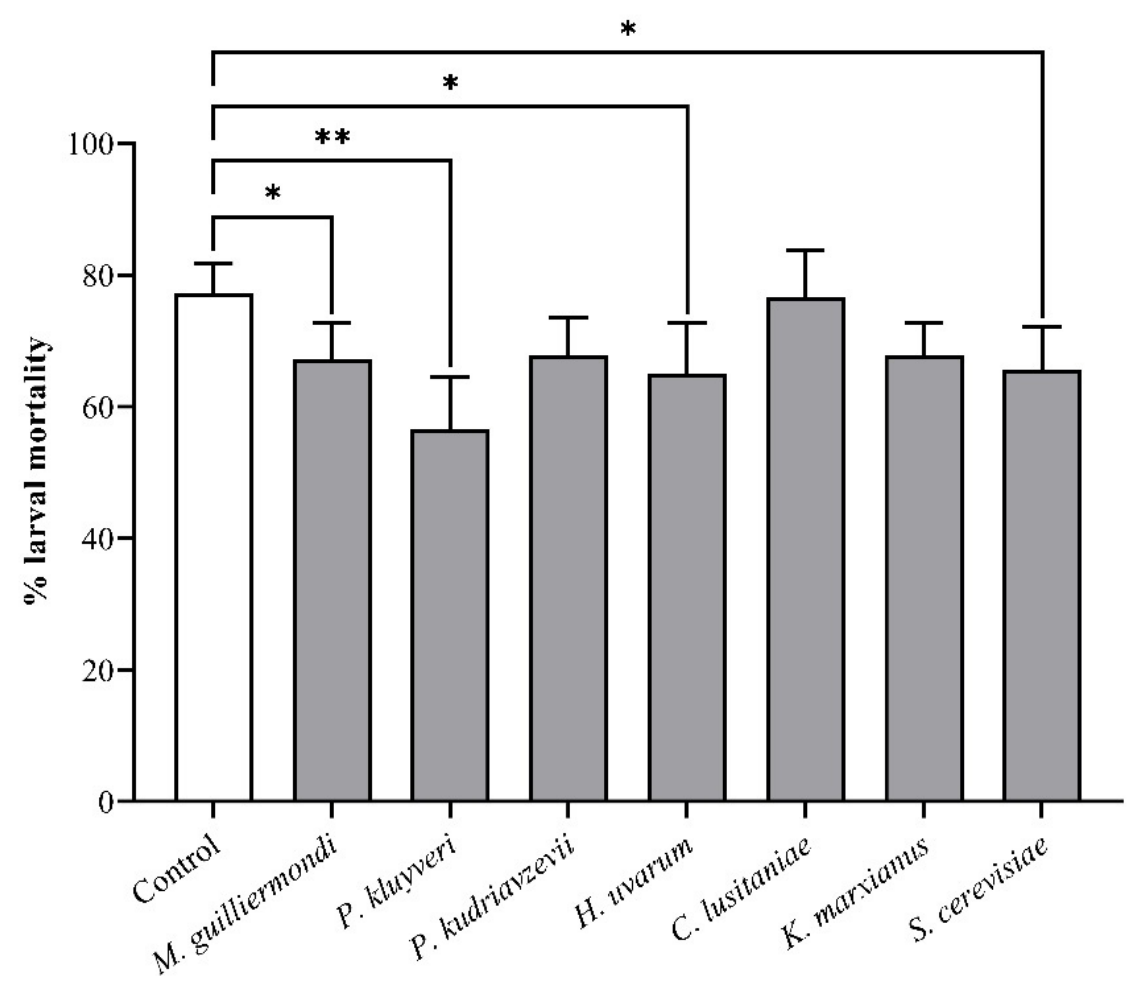

Figure 2. The mortality of T. leucotreta larvae, determined after $35 \mathrm{~d}$ on sterilised Navel oranges $(n=36)$, inoculated with yeast or left untreated. Data are shown as mean $\pm \mathrm{SE}$. ${ }^{*}$ and ${ }^{* *}$ indicate significant differences from the control according to a Fisher's exact test ( $p<0.05$ and $p<0.01$, respectively).

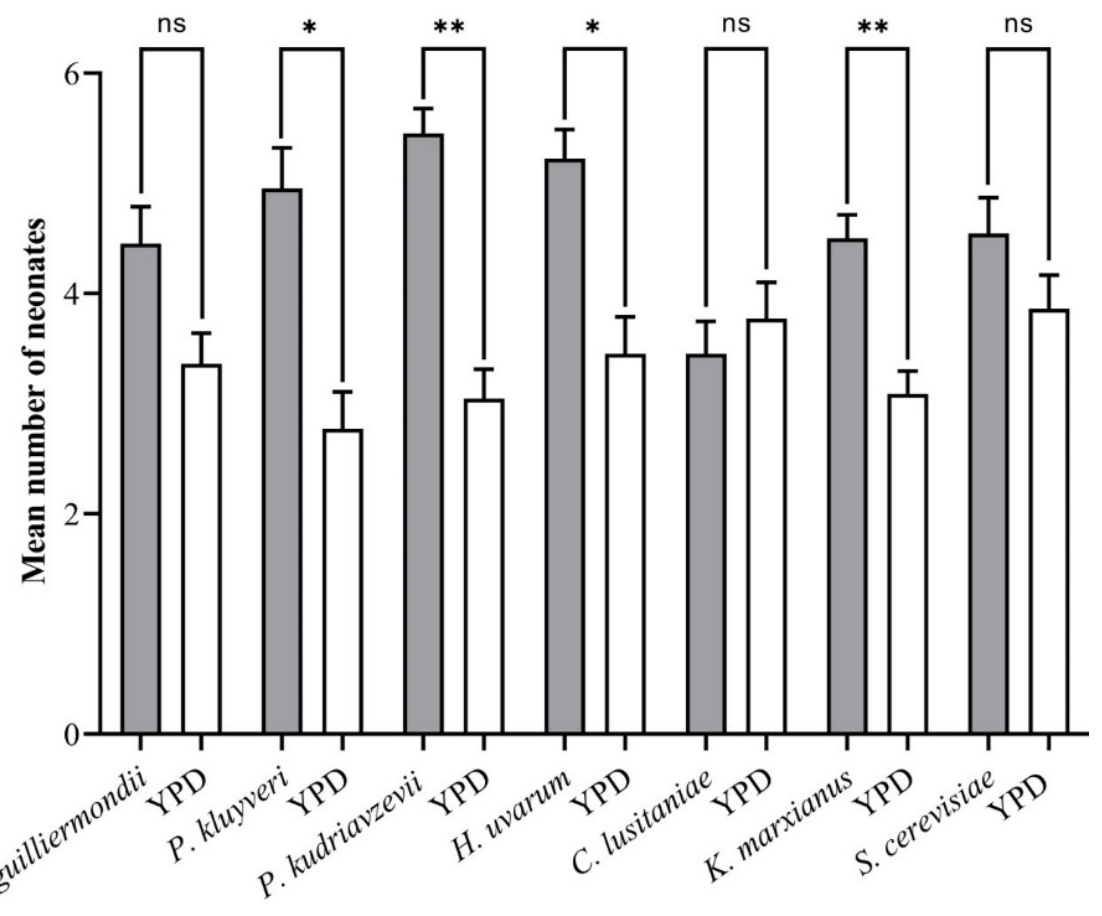

Figure 3. The attraction and feeding of neonate T. leucotreta in response to yeasts $(n=22) .{ }^{*}$ and ** indicate significant differences from the control (YPD) according to a Student's $t$-test $(p<0.05$ and $p<0.01$, respectively); ns indicates that differences were not significant. 


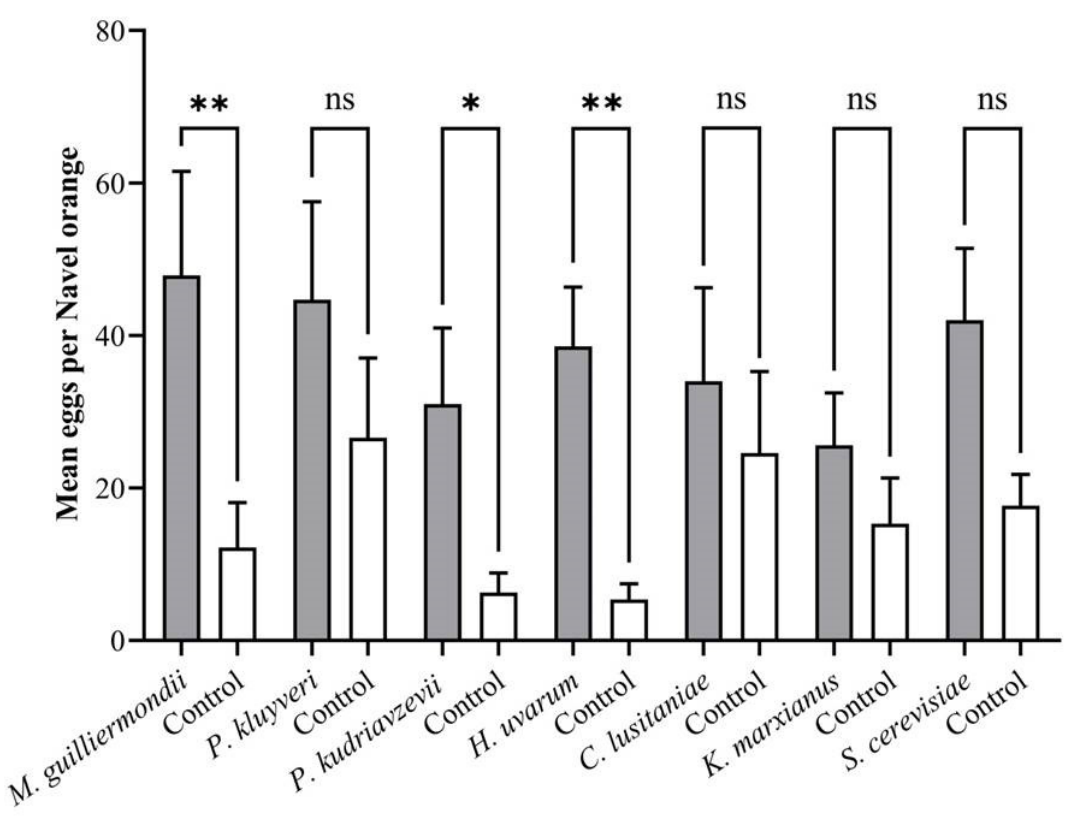

Figure 4. The oviposition preference of adult T. leucotreta females for yeast during two-choice tests $(n=10) .{ }^{*}$ and ${ }^{* *}$ indicate significant differences from the control according to a Student's $t$-test $(p<0.05$ and $p<0.01$, respectively); ns indicates that differences were not significant.

\section{Discussion}

Most interactions between insects and microorganisms have been studied for their potential use as insect pathogens and not for their role as mutualists [43]. Yeasts play an essential role in the nutrition physiology and host attraction of many insects [19]. The volatile profiles produced by yeasts have also been shown to elicit strong behavioural responses in both lepidopteran larvae and adults $[26,41,44]$. The potential value of incorporating mutualistic yeast into current strategies to control insect pests has only recently been explored, leading to new pest management strategies [27-29]. As this is still a developing area of research, little is known about the influence of naturally occurring yeasts on T. leucotreta.

Meyerozyma guilliermondii, $P$. kudriavzevii, $P$. kluyveri and H. uvarum are widely distributed in the environment and often associated with citrus fruits or fermented food [45-48], so their association with T. leucotreta is not unexpected. Hanseniaspora uvarum is also thought to have a mutualistic relationship with Drosophila suzukii (Matsumura) (Diptera: Drosophilidae), a significant pest of soft summer fruits elsewhere in the world [49-52]. Larval development assays demonstrated that the isolated yeasts have a beneficial effect on T. leucotreta larvae by reducing their development periods and mortality. There is growing support that the vast majority of insects feed and benefit from yeast in their diet. Yeast multi-cultures have been shown to improve insect development [19]. The composition of an insect's diet has been shown to affect their adult life traits, including food preference and host locating $[53,54]$. During larval development, a limited yeast supply can negatively affect adult food preference, copulation, fecundity and longevity $[14,53]$. In Drosophila species, larval diets containing mixed yeast cultures provide more significant development speed and survivability benefits than single yeast cultures [55]. The yeast diet of juvenile insects directly influences their fitness and development and indirectly affects adult life traits $[14,53]$.

Detrimental fungal infections were observed less frequently on Navel oranges treated with yeast than those without, during larval development assays. Kluyveromyces marxianus has been reported to be an effective biological control agent against Penicillium digitatum, a fungus that causes green mould in citrus fruit, in combination with sodium bicarbonate $\left(\mathrm{NaHCO}_{3}\right)$ [56]. Hanseniaspora uvarum, in combination with phosphatidylcholine, has also shown great potential as a biological control agent against $P$. digitatum [57]. Meyerozyma guilliermondii has also exhibited 
potential as a biological control agent against fungi responsible for postharvest spoilage of fruit and vegetables [58]. Thaumatotibia leucotreta-infested fruit often do not rot, or rotting is delayed, particularly when compared to Ceratitis capitata (Wiedemann) (Diptera: Tephritidae) (Mediterranean fruit fly) infestations, indicating that T. leucotreta larvae may feed on secondary fungal infections, preventing, or delaying, the fruit's decay. A similar observation has been made in the case of $C$. pomonella larval feeding galleries, which rarely become infested with mould [26]. This stands to reason, as the standard artificial diet for laboratory rearing of T. leucotreta used to be a Mucor or Rhizopus fungal species-inoculated maise meal diet $[59,60]$.

Integrated pest management programmes in South Africa successfully use both semiochemicals and pathogens to manage T. leucotreta populations [33,36,61]. Combining semiochemicals with pathogens in attract-and-kill strategies could significantly increase the efficacy of currently used biological control agents $[62,63]$. The behaviour of neonate T. leucotreta was influenced by H. uvarum, P. kluyveri, P. kudriavzevii and K. marxianus. Volatiles produced by yeasts are thought to stimulate larval feeding [27]. Insect larvae have been shown to prefer specific yeast species over others during the early stages of development $[54,64]$. In larval feeding assays, it has been demonstrated that Spodoptera littoralis (Boisduval) (Lepidoptera, Noctuidae) larvae preferred phyllosphere yeasts over yeasts associated with fruit and frugivorous insects [41]. Increasing larval feeding with yeast increases the probability that a simultaneously applied biopesticide will be ingested by the insect [27-29].

Meyerozyma guilliermondii, P. kudriavzevii and H. uvarum were shown to significantly affect adult T. leucotreta females' oviposition preference. Both H. uvarum and P. kudriavzevii have now been shown to influence T. leucotreta neonate and adult female behaviour. They are known to be associated with citrus; thus, their influence on T. leucotreta is not unexpected. To date, the behavioural influences of these yeasts on lepidopteran moths have not been well documented. However, their influence on other agriculturally important pests has been reported. Hanseniaspora uvarum has been shown to affect the ovipositional behaviour of drosophilid flies, acting as both an attractant and repellant [50,65,66]. Using H. uvarum as a live yeast bait for a selective lure against $D$. suzukii has been suggested [67]. The odour profiles of host plants are comprised of numerous volatile organic compounds in a specific blend ratio [68]. Microorganisms can modify this odour profile produced by plants [44]. Moreover, the metabolites produced by gut-associated microbes can affect insects foraging and oviposition behaviour [25]. Volatiles produced by yeasts can elicit a strong response in neonates and adult insects $[14,26,41,53]$. Host finding and discrimination for C. pomonella are mediated by the yeasts' volatile signatures [26]. In contrast to vision and contact, olfactory cues can be detected at a considerable distance from the source [66,69].

Six yeast species were successfully isolated and identified from $T$. leucotreta larvae collected from geographically distinct citrus-producing regions. Larval feeding and oviposition assays demonstrated that the isolated yeast species elicit behavioural responses in $T$. leucotreta neonates and adult females. The results generated from this work may help further the development of environmentally safe semiochemicals for population monitoring and control of T. leucotreta in South Africa. Future work arising from this study would involve conducting detached fruit bioassays and semi-field trials to evaluate the efficacy of a combination with biological control agents currently used against $T$. leucotreta.

Supplementary Materials: The following are available online at https:/ / www.mdpi.com/article/ 10.3390 /insects13030243/s1, Figure S1: Feeding response of neonate T. leucotreta $(n=29)$ to YPD medium containing either $(\mathrm{A})$ red or $(\mathrm{B})$ blue food colourant $(P>0.05 ; \mathrm{t}=0.2133 ; \mathrm{df}=27$ (Students $t$ test)). Images captured by David Taylor, Table S1: Oligonucleotides used to amplify and sequence the internal transcribed spacer (ITS) region and D1/D2 domain of large subunit (LSU) on yeast genomes. 


\begin{abstract}
Author Contributions: Conceptualisation, S.D.M., M.P.H. and C.K.; methodology, S.D.M., M.v.d.M. and M.D.J.; formal analysis, M.v.d.M. and M.D.J.; investigation, M.v.d.M.; resources, S.D.M., M.P.H., C.K. and M.D.J.; data curation, M.v.d.M.; writing—original draft preparation, M.v.d.M.; writing—review and editing, M.v.d.M., M.D.J., C.K., M.P.H. and S.D.M.; visualisation, M.v.d.M.; supervision, C.K., M.P.H. and S.D.M.; project administration, M.v.d.M., M.P.H., S.D.M. and C.K.; funding acquisition, M.P.H. and S.D.M. All authors have read and agreed to the published version of the manuscript.

Funding: The authors would like to thank Citrus Research International (CRI), Research for Citrus Exports (RCE) Programme of the Department of Science and Innovation (DSI), the Research Council of Rhodes University for financial support. The South African Research Chairs Initiative of the Department of Science and Technology and the National Research Foundation (NRF) of South Africa (grant code 84643) provided additional funding for this work.
\end{abstract}

Institutional Review Board Statement: Not applicable.

Informed Consent Statement: Not applicable.

Data Availability Statement: The data presented in this study are available on request from the corresponding author.

Acknowledgments: Special thanks must be given to Candice Coombes for maintaining the T. leucotreta colony held at Rhodes University, which was used for the duration of this research.

Conflicts of Interest: The authors declare no conflict of interest.

\title{
References
}

1. Hosokawa, T.; Fukatsu, T. Relevance of microbial symbiosis to insect behavior. Curr. Opin. Insect Sci. 2020, 39, 91-100. [CrossRef]

2. Douglas, A.E. Multiorganismal Insects: Diversity and Function of Resident Microorganisms. Annu. Rev. Entomol. 2015, 60, 17-34. [CrossRef] [PubMed]

3. Engel, P.; Moran, N.A. The gut microbiota of insects-Diversity in structure and function. FEMS Microbiol. Rev. 2013, 37, 699-735. [CrossRef]

4. Gupta, A.; Nair, S. Dynamics of Insect-Microbiome Interaction Influence Host and Microbial Symbiont. Front. Microbiol. 2020, 11, 1357. [CrossRef] [PubMed]

5. Becher, P.G.; Hagman, A.; Verschut, V.; Chakraborty, A.; Rozpędowska, E.; Lebreton, S.; Bengtsson, M.; Flick, G.; Witzgall, P.; Piškur, J. Chemical signaling and insect attraction is a conserved trait in yeasts. Ecol. Evol. 2018, 8, 2962-2974. [CrossRef]

6. Madden, A.A.; Epps, M.J.; Fukami, T.; Irwin, R.E.; Sheppard, J.; Sorger, D.M.; Dunn, R.R. The ecology of insect-yeast relationships and its relevance to human industry. Proc. R. Soc. B Biol. Sci. 2018, 285, 20172733. [CrossRef]

7. Douglas, A.E. The Molecular Basis of Bacterial-Insect Symbiosis. J. Mol. Biol. 2014, 426, 3830-3837. [CrossRef] [PubMed]

8. Martino, M.E.; Ma, D.; Leulier, F. Microbial influence on Drosophila biology. Curr. Opin. Microbiol. 2017, 38, 165-170. [CrossRef]

9. Gurung, K.; Wertheim, B.; Salles, J.F. The microbiome of pest insects: It is not just bacteria. Entomol. Exp. Appl. 2019, 167, 156-170. [CrossRef]

10. Wang, S.; Wang, L.; Fan, X.; Yu, C.; Feng, L.; Yi, L. An Insight into Diversity and Functionalities of Gut Microbiota in Insects Curr. Microbiol. 2020, 77, 1976-1986. [CrossRef] [PubMed]

11. Barraclough, T.G. How Do Species Interactions Affect Evolutionary Dynamics Across Whole Communities? Annu. Rev. Ecol. Evol. Syst. 2015, 46, 25-48. [CrossRef]

12. Chandler, D.; Bailey, A.S.; Tatchell, G.M.; Davidson, G.; Greaves, J.; Grant, W.P. The development, regulation and use of biopesticides for integrated pest management. Philos. Trans. R. Soc. B Biol. Sci. 2011, 366, 1987-1998. [CrossRef] [PubMed]

13. McFall-Ngai, M.; Hadfield, M.G.; Bosch, T.C.G.; Carey, H.V.; Domazet-Lošo, T.; Douglas, A.E.; Dubilier, N.; Eberl, G.; Fukami, T.; Gilbert, S.F.; et al. Animals in a bacterial world, a new imperative for the life sciences. Proc. Natl. Acad. Sci. USA 2013, 110, 3229-3236. [CrossRef]

14. Murgier, J.; Everaerts, C.; Farine, J.-P.; Ferveur, J.-F. Live yeast in juvenile diet induces species-specific effects on Drosophila adult behaviour and fitness. Sci. Rep. 2019, 9, 8873. [CrossRef] [PubMed]

15. Yun, J.-H.; Roh, S.W.; Whon, T.W.; Jung, M.-J.; Kim, M.-S.; Park, D.-S.; Yoon, C.; Nam, Y.-D.; Kim, Y.-J.; Choi, J.-H.; et al. Insect Gut Bacterial Diversity Determined by Environmental Habitat, Diet, Developmental Stage, and Phylogeny of Host. Appl. Environ. Microbiol. 2014, 80, 5254-5264. [CrossRef]

16. MsangoSoko, K.; Gandotra, S.; Chandel, R.K.; Sharma, K.; Ramakrishinan, B.; Subramanian, A.S. Composition and Diversity of Gut Bacteria Associated with the Eri Silk Moth, Samia ricini, (Lepidoptera: Saturniidae) as Revealed by Culture-Dependent and Metagenomics Analysis. J. Microbiol. Biotechnol. 2020, 30, 1367-1378. [CrossRef]

17. Paniagua Voirol, L.R.; Frago, E.; Kaltenpoth, M.; Hilker, M.; Fatouros, N.E. Bacterial Symbionts in Lepidoptera: Their Diversity, Transmission, and Impact on the Host. Front. Microbiol. 2018, 9, 556. [CrossRef] 
18. Sree, K.S.; Varma, A. (Eds.) Biocontrol of Lepidopteran Pests: Use of Soil Microbes and Their Metabolites; Soil Biology; Springer International Publishing: Cham, Switzerland, 2015, ISBN 978-3-319-14498-6.

19. Stefanini, I. Yeast-insect associations: It takes guts. Yeast 2018, 35, 315-330. [CrossRef]

20. Zchori-Fein, E.; Bourtzis, K. Manipulative Tenants: Bacteria Associated with Arthropods; CRC Press: Boca Raton, FL, USA, 2011, ISBN 978-1-4398-2749-9.

21. Günther, C.S.; Goddard, M.R. Do yeasts and Drosophila interact just by chance? Fungal Ecol. 2019, 38, 37-43. [CrossRef]

22. Bellutti, N.; Gallmetzer, A.; Innerebner, G.; Schmidt, S.; Zelger, R.; Koschier, E.H. Dietary yeast affects preference and performance in Drosophila suzukii. J. Pest Sci. 2017, 91, 651-660. [CrossRef]

23. Spitaler, U.; Bianchi, F.; Eisenstecken, D.; Castellan, I.; Angeli, S.; Dordevic, N.; Robatscher, P.; Vogel, R.F.; Koschier, E.H.; Schmidt, S. Yeast species affects feeding and fitness of Drosophila suzukii adults. J. Pest Sci. 2020, 93, 1295-1309. [CrossRef]

24. Becher, P.G.; Flick, G.; Rozpędowska, E.; Schmidt, A.; Hagman, A.; Lebreton, S.; Larsson, M.C.; Hansson, B.S.; Piškur, J.; Witzgall, P.; et al. Yeast, not fruit volatiles mediate Drosophila melanogaster attraction, oviposition and development. Funct. Ecol. 2012, 26, 822-828. [CrossRef]

25. Grunseich, J.M.; Thompson, M.N.; Aguirre, N.M.; Helms, A.M. The Role of Plant-Associated Microbes in Mediating Host-Plant Selection by Insect Herbivores. Plants 2020, 9, 6. [CrossRef] [PubMed]

26. Witzgall, P.; Proffit, M.; Rozpedowska, E.; Becher, P.G.; Andreadis, S.; Coracini, M.; Lindblom, T.; Ream, L.J.; Hagman, A.; Bengtsson, M.; et al. "This is not an Apple"-Yeast Mutualism in Codling Moth. J. Chem. Ecol. 2012, 38, 949-957. [CrossRef]

27. Knight, A.L.; Witzgall, P. Combining Mutualistic Yeast and Pathogenic Virus-A Novel Method for Codling Moth Control. J. Chem. Ecol. 2013, 39, 1019-1026. [CrossRef]

28. Knight, A.L.; Basoalto, E.; Witzgall, P. Improving the Performance of the Granulosis Virus of Codling Moth (Lepidoptera: Tortricidae) by Adding the Yeast Saccharomyces cerevisiae with Sugar. Environ. Entomol. 2015, 44, 252-259. [CrossRef]

29. Knight, A.L.; Basoalto, E.; Yee, W.; Hilton, R.; Kurtzman, C.P. Adding yeasts with sugar to increase the number of effective insecticide classes to manage Drosophila suzukii (Matsumura) (Diptera: Drosophilidae) in cherry: Adding Yeast and Sugar to Manage Spotted-Wing Drosophila. Pest Manag. Sci. 2016, 72, 1482-1490. [CrossRef]

30. Bedford, E.C.G.; Van den Berg, M.A.; De Villiers, E.A. Citrus Pests in the Republic of South Africa; Institute for Tropical and Subtropical Crops; Outspan International: Nelspruit, South Africa, 1998, ISBN 978-0-620-22031-6.

31. Hattingh, V.; Moore, S.; Kirkman, W.; Goddard, M.; Thackeray, S.; Peyper, M.; Sharp, G.; Cronjé, P.; Pringle, K. An Improved Systems Approach as a Phytosanitary Measure for Thaumatotibia leucotreta (Lepidoptera: Tortricidae) in Export Citrus Fruit from South Africa. J. Econ. Entomol. 2020, 113, 700-711. [CrossRef]

32. Moore, S.D.; Grout, T.G.; Hattingh, V.; Hofmeyr, J.H. Thresholds and Guidelines for Intervention against Citrus Pests. S. Afr. Fruit J. 2008, 7, 77-81.

33. Moore, S.D. Biological Control of a Phytosanitary Pest (Thaumatotibia leucotreta): A Case Study. Int. J. Environ. Res. Public Health 2021, 18, 1198. [CrossRef]

34. Mkiga, A.M.; Mohamed, S.A.; Du Plessis, H.; Khamis, F.M.; Ekesi, S. Field and Laboratory Performance of False Codling Moth, Thaumatotibia leucotreta (Lepidoptera: Troticidae) on Orange and Selected Vegetables. Insects 2019, 10, 63. [CrossRef] [PubMed]

35. Hatting, J.L.; Moore, S.D.; Malan, A.P. Microbial control of phytophagous invertebrate pests in South Africa: Current status and future prospects. J. Invertebr. Pathol. 2019, 165, 54-66. [CrossRef] [PubMed]

36. Moore, S.D.; Kirkman, W.; Richards, G.I.; Stephen, P.R. The Cryptophlebia leucotreta Granulovirus-10 Years of Commercial Field Use. Viruses 2015, 7, 1284-1312. [CrossRef] [PubMed]

37. Pincus, D.H.; Orenga, S.; Chatellier, S. Yeast identification-Past, present, and future methods. Med. Mycol. 2007, 45, 97-121. [CrossRef]

38. Kumar, S.; Stecher, G.; Li, M.; Knyaz, C.; Tamura, K. MEGA X: Molecular Evolutionary Genetics Analysis across Computing Platforms. Mol. Biol. Evol. 2018, 35, 1547-1549. [CrossRef]

39. Opoku-Debrah, J.K. Studies on Existing and New Isolates of Cryptophlebia leucotreta Granulovirus (CrleGV) on Thaumatotibia leucotreta Populations from a Range of Geographic Regions in South Africa. Ph.D. Thesis, Rhodes University, Makhanda, South Africa, 2012.

40. Moore, S.D.; Richards, G.; Chambers, C.; Hendry, D.A. An Improved Larval Diet for Commercial Mass Rearing of the False Codling Moth, Thaumatotibia leucotreta (Meyrick) (Lepidoptera: Tortricidae). Afr. Entomol. 2014, 22, 216-219. [CrossRef]

41. Ljunggren, J.; Borrero-Echeverry, F.; Chakraborty, A.; Lindblom, T.U.T.; Hedenström, E.; Karlsson, M.; Witzgall, P.; Bengtsson, M. Yeast Volatomes Differentially Affect Larval Feeding in an Insect Herbivore. Appl. Environ. Microbiol. 2019, 85, e01761-19. [CrossRef]

42. Love, C.N.; Hill, M.; Moore, S.D. Thaumatotibia leucotreta and the Navel orange: Ovipositional preferences and host susceptibility. J. Appl. Entomol. 2014, 138, 600-611. [CrossRef]

43. Hajek, A.E.; McManus, M.L.; Delalibera, I. A review of introductions of pathogens and nematodes for classical biological control of insects and mites. Biol. Control 2007, 41, 1-13. [CrossRef]

44. Davis, T.S.; Crippen, T.L.; Hofstetter, R.W.; Tomberlin, J.K. Microbial Volatile Emissions as Insect Semiochemicals. J. Chem. Ecol. 2013, 39, 840-859. [CrossRef] 
45. Heras-Vazquez, F.J.L.; Mingorance-Cazorla, L.; Clemente-Jimenez, J.M.; Rodriguez-Vico, F. Identification of yeast species from orange fruit and juice by RFLP and sequence analysis of the 5.8S rRNA gene and the two internal transcribed spacers. FEMS Yeast Res. 2003, 3, 3-9. [CrossRef] [PubMed]

46. Vadkertiová, R.; Molnárová, J.; Vránová, D.; Sláviková, E. Yeasts and yeast-like organisms associated with fruits and blossoms of different fruit trees. Can. J. Microbiol. 2012, 58, 1344-1352. [CrossRef] [PubMed]

47. Hidalgo, C.; Mateo, E.; Mas, A.; Torija, M. Identification of yeast and acetic acid bacteria isolated from the fermentation and acetification of persimmon (Diospyros kaki). Food Microbiol. 2012, 30, 98-104. [CrossRef] [PubMed]

48. Ganter, P.F.; Morais, P.B.; Rosa, C.A. Yeasts in Cacti and Tropical Fruit. In Yeasts in Natural Ecosystems: Diversity; Buzzini, P., Lachance, M.-A., Yurkov, A., Eds.; Springer International Publishing: Cham, Switzerland, 2017; pp. 225-264, ISBN 978-3-31962683-3.

49. Bellamy, D.E.; Sisterson, M.S.; Walse, S.S. Quantifying Host Potentials: Indexing Postharvest Fresh Fruits for Spotted Wing Drosophila, Drosophila suzukii. PLoS ONE 2013, 8, e61227. [CrossRef] [PubMed]

50. Hamby, K.A.; Hernández, A.; Boundy-Mills, K.; Zalom, F.G. Associations of Yeasts with Spotted-Wing Drosophila (Drosophila suzukii; Diptera: Drosophilidae) in Cherries and Raspberries. Appl. Environ. Microbiol. 2012, 78, 4869-4873. [CrossRef]

51. Lee, J.C.; Bruck, D.J.; Curry, H.; Edwards, D.; Haviland, D.R.; Van Steenwyk, R.A.; Yorgey, B.M. The susceptibility of small fruits and cherries to the spotted-wing drosophila, Drosophila suzukii. Pest Manag. Sci. 2011, 67, 1358-1367. [CrossRef]

52. Wang, X.-G.; Stewart, T.J.; Biondi, A.; Chavez, B.A.; Ingels, C.; Caprile, J.; Grant, J.A.; Walton, V.M.; Daane, K.M. Population dynamics and ecology of Drosophila suzukii in Central California. J. Pest Sci. 2016, 89, 701-712. [CrossRef]

53. Grangeteau, C.; Yahou, F.; Everaerts, C.; Dupont, S.; Farine, J.-P.; Beney, L.; Ferveur, J.-F. Yeast quality in juvenile diet affects Drosophila melanogaster adult life traits. Sci. Rep. 2018, 8, 13070. [CrossRef]

54. Lewis, M.T.; Hamby, K.A. Differential Impacts of Yeasts on Feeding Behavior and Development in Larval Drosophila suzukii (Diptera:Drosophilidae). Sci. Rep. 2019, 9, 13370. [CrossRef]

55. Starmer, W.T.; Aberdeen, V. The Nutritional Importance of Pure and Mixed Cultures of Yeasts in the Development of Drosophila mulleri Larvae in Opuntia Tissues and Its Relationship to Host Plant Shifts. In Ecological and Evolutionary Genetics of Drosophila; Springer: New York, NY, USA, 1990; pp. 145-160.

56. Geng, P.; Chen, S.; Hu, M.; Rizwan-Ul-Haq, M.; Lai, K.; Qu, F.; Zhang, Y. Combination of Kluyveromyces marxianus and sodium bicarbonate for controlling green mold of citrus fruit. Int. J. Food Microbiol. 2011, 151, 190-194. [CrossRef]

57. Li, W.; Zhang, H.; Li, P.; Apaliya, M.T.; Yang, Q.; Peng, Y.; Zhang, X. Biocontrol of postharvest green mold of oranges by Hanseniaspora uvarum Y3 in combination with phosphatidylcholine. Biol. Control 2016, 103, 30-38. [CrossRef]

58. Lima, J.R.; Gondim, D.M.F.; Oliveira, J.T.A.; Oliveira, F.S.A.; Goncalves, L.R.B.; Viana, F.M.P. Use of killer yeast in the management of postharvest papaya anthracnose. Postharvest Biol. Technol. 2013, 83, 58-64. [CrossRef]

59. Ripley, L.B.; Hepburn, G.A.; Dick, J. Mass Breeding of False Codling-Moth, Argyroploce leucotreta Meyr., in Artificial Media; Science Bulletin of the Department of Agriculture and Forestry of the Union of South Africa: Pretoria, South Africa, 1939.

60. Theron, P.P.A. Studies in Verband met die Verskaffing van Gashere vir die Massateelt van Appelmotparasiete; Staatsdrukker: Pretoria, South Africa, 1948.

61. Malan, A.P.; Von Diest, J.; Moore, S.D.; Addison, P. Control Options for False Codling Moth, Thaumatotibia leucotreta (Lepidoptera: Tortricidae), in South Africa, with Emphasis on the Potential Use of Entomopathogenic Nematodes and Fungi. Afr. Entomol. 2018, 26, 14-29. [CrossRef]

62. Gregg, P.C.; Del Socorro, A.P.; Landolt, P.J. Advances in Attract-and-Kill for Agricultural Pests: Beyond Pheromones. Annu. Rev. Entomol. 2018, 63, 453-470. [CrossRef] [PubMed]

63. El-Sayed, A.M.; Suckling, D.; Byers, J.A.; Jang, E.B.; Wearing, C.H. Potential of "Lure and Kill" in Long-Term Pest Management and Eradication of Invasive Species. J. Econ. Entomol. 2009, 102, 815-835. [CrossRef] [PubMed]

64. Anagnostou, C.; Dorsch, M.; Rohlfs, M. Influence of dietary yeasts on Drosophila melanogaster life-history traits. Entomol. Exp. Appl. 2010, 136, 1-11. [CrossRef]

65. Piper, A.M.; Farnier, K.; Linder, T.; Speight, R.; Cunningham, J.P. Two Gut-Associated Yeasts in a Tephritid Fruit Fly have Contrasting Effects on Adult Attraction and Larval Survival. J. Chem. Ecol. 2017, 43, 891-901. [CrossRef] [PubMed]

66. Tasin, M.; Herrera, S.L.; Knight, A.L.; Barros-Parada, W.; Fuentes-Contreras, E.; Pertot, I. Volatiles of Grape Inoculated with Microorganisms: Modulation of Grapevine Moth Oviposition and Field Attraction. Microb. Ecol. 2018, 76, 751-761. [CrossRef]

67. Hamby, K.A.; Becher, P.G. Current knowledge of interactions between Drosophila suzukii and microbes, and their potential utility for pest management. J. Pest Sci. 2016, 89, 621-630. [CrossRef]

68. Knudsen, J.T.; Tollsten, L.; Bergström, L.G. Floral scents-A checklist of volatile compounds isolated by head-space techniques Phytochemistry 1993, 33, 253-280. [CrossRef]

69. Bruce, T.J.A.; Wadhams, L.J.; Woodcock, C.M. Insect host location: A volatile situation. Trends Plant Sci. 2005, 10, 269-274 [CrossRef] [PubMed] 